\title{
Fully Consistent Extensions of Partially Defined Boolean Functions with Missing Bits ${ }^{\star}$
}

\author{
Endre Boros ${ }^{1}$, Toshihide Ibaraki $^{2}$, and Kazuhisa Makino ${ }^{3}$ \\ 1 RUTCOR, Rutgers University, 640 Bartholomew Road, Piscataway, NJ \\ 08854-8003, USA. boros@rutcor.rutgers.edu \\ 2 Department of Applied Mathematics and Physics, Graduate School of Informatics, \\ Kyoto University, Kyoto 606-8501, Japan. ibaraki@i.kyoto-u.ac.jp \\ 3 Department of Systems and Human Science, Graduate School of Engineering \\ Science, Osaka University, Toyonaka, Osaka 560-8531, Japan. \\ makino@sys.es.osaka-u.ac.jp
}

\begin{abstract}
In this paper we consider four different definitions for an extension of a partially defined Boolean function in which the input contains some missing bits. We show that, for many general and reasonable families of function classes, three of these extensions are mathematically equivalent. However we also demonstrate that such an equivalence does not hold for all classes.
\end{abstract}

\section{Introduction}

A Boolean function, or a function in short, is a mapping $f: \mathbb{B}^{n} \mapsto \mathbb{B}$, where $\mathbb{B}=\{0,1\}$. Given a function $f$, a Boolean vector $x \in \mathbb{B}^{n}$ is called its true vector, if $f(x)=1$, and its false vector, if $f(x)=0$. Let us denote the set of true vectors of $f$ by $T(f)$, and let $F(f)=\mathbb{B}^{n} \backslash T(f)$ denote the set of its false vectors. Let us denote by $\mathcal{C}_{\text {all }}$ the family of all Boolean functions $f: \mathbb{B}^{n} \mapsto \mathbb{B}$, and let us call any subfamily of $\mathcal{C}_{\text {all }}$ a class. We shall consider various classes of Boolean functions in the sequel, defined in many different ways.

A partially defined Boolean function (a $p d B f$ in short) is defined by a pair of sets $(T, F)$ such that $T, F \subseteq \mathbb{B}^{n}$. A Boolean function $f$ is called an extension of the pdBf $(T, F)$ if $T \subseteq T(f)$ and $F \subseteq F(f)$ hold, that is, if such an $f$ correctly classifies all the vectors $a \in T$ and $b \in F$. Let us denote by $\mathcal{E}(T, F)$ the family of extensions of the pdBf $(T, F)$. Evidently, the disjointness of the sets $T$ and $F$ is a necessary and sufficient condition for the existence of an extension $\mathcal{E}(T, F) \neq \emptyset$. It may not be evident, however, to find out if a given pdBf has an extension belonging to a particular class $\mathcal{C}$ of Boolean functions, or not. This problem has been studied in various fields such as learning theory, knowledge discovery, data mining and logical analysis of data [145681113.

* This research was partially supported by ONR (Grant N00014-92-J-1375), DARPA (Contract Number N66001-97-C-8537), and the Scientific Grants in Aid by the Ministry of Education, Science, Sports and Culture of Japan. The visit of the first author to Kyoto University was made possible by the grant (09044160) of the Ministry of Education, Science, Sports and Culture of Japan. 
In practical cases, the fact that $\mathcal{C} \cap \mathcal{E}(T, F)=\emptyset$ might be due to some classification errors in the input. To correct this type of errors, provided that they are not in a large number, one can consider the optimization problem of finding the largest subsets $T^{*} \subseteq T$ and $F^{*} \subseteq F$ for which $\mathcal{E}\left(T^{*}, F^{*}\right) \cap \mathcal{C} \neq \emptyset$ holds. These problems have extensively been studied (e.g., in 811]) for a large variety of classes.

In this paper we shall consider another type of errors in the input, the case in which some data vectors are "incomplete" in the sense that some of their components are not available at the time of reading the input. Such missing information may either be due to some measurement errors at some earlier stages of data generation, or they are the results of data entry errors, or such lack of information might be due to the high cost of obtaining those.

To model such situations, let us consider the set $\mathbb{M}=\{0,1, *\}$, and let us interpret the asterisk components $*$ of a vector $v \in \mathbb{M}^{n}$ as missing bits. Then, a $p d B f$ with missing bits (or in short a $p B m b$ ) can be defined as a pair $(\tilde{T}, \tilde{F})$, where $\tilde{T}, \tilde{F} \subseteq \mathbb{M}^{n}$. Given a pBmb, it is possible to consider more than one notion of extensions $f$, depending on how to interpret *'s in the extensions; in this paper, we give four different definitions, two of which have already been discussed in [9]. We then prove for many important classes of functions that three of these definitions are equivalent. However, it is also demonstrated that this equivalence does not hold for all classes.

\section{Extensions of $\mathrm{pBmbs}$}

For a vector $v \in \mathbb{M}^{n}$, let us introduce the notations $O N(v)=\left\{j \mid v_{j}=1, j=\right.$ $1,2, \ldots, n\}$ and $O F F(v)=\left\{j \mid v_{j}=0, j=1,2, \ldots, n\right\}$. For a subset $\tilde{A} \subseteq \mathbb{M}^{n}$, let $S(\tilde{A})=\left\{(v, j) \mid v \in \tilde{A}, v_{j}=*\right\}$ be the collection of all missing bits of the vectors in $\tilde{A}$. If $\tilde{A}$ is a singleton $\{v\}$, we shall also write $S(v)$ instead of $S(\{v\})$. Clearly, $\mathbb{B}^{n} \subseteq \mathbb{M}^{n}$, and $v \in \mathbb{B}^{n}$ holds if and only if $S(v)=\emptyset$. Let us consider a binary assignment $\alpha \in \mathbb{B}^{Q}$ to a subset $Q \subseteq S(\tilde{A})$ of the missing bits. Then $v^{\alpha}$ denotes the vector obtained from $v \in \tilde{A}$ by replacing the $*$ components which belong to $Q$ by the binary values assigned by $\alpha$ :

$$
v_{j}^{\alpha}= \begin{cases}v_{j} & \text { if }(v, j) \notin Q \\ \alpha(v, j) & \text { if }(v, j) \in Q .\end{cases}
$$

Let $\tilde{A}^{\alpha}$ denote the set $\left\{v^{\alpha} \mid v \in \tilde{A}\right\}$. For example, for the set $\tilde{A}=\{u=(1, *, 0,1)$, $v=(0,1, *, *), w=(1,1, *, 0)\} \subset \mathbb{M}^{4}$ we have $S(\tilde{A})=\{(u, 2),(v, 3),(v, 4)$, $(w, 3)\}$. If $Q=\{(u, 2),(v, 4)\}$, an assignment $(\alpha(u, 2), \alpha(v, 4))=(1,0) \in \mathbb{B}^{Q}$ yields $\tilde{A}^{\alpha}=\left\{u^{\alpha}=(1,1,0,1), v^{\alpha}=(0,1, *, 0), w^{\alpha}=(1,1, *, 0)\right\}$.

To a pBmb $(\tilde{T}, \tilde{F})$ we shall always associate the set $S=S(\tilde{T} \cup \tilde{F})$ of its missing bits. For a pBmb $(\tilde{T}, \tilde{F})$ and an assignment $\alpha \in \mathbb{B}^{S}$, let $\left(\tilde{T}^{\alpha}, \tilde{F}^{\alpha}\right)$ be the pdBf defined by $\tilde{T}^{\alpha}=\left\{a^{\alpha} \mid a \in \tilde{T}\right\}$ and $\tilde{F}^{\alpha}=\left\{b^{\alpha} \mid b \in \tilde{F}\right\}$.

Let us call a pBmb $(\tilde{T}, \tilde{F})$ consistent with respect to a class $\mathcal{C}$ of Boolean functions, if there exists an assignment $\alpha \in \mathbb{B}^{S}$ for which the $\operatorname{pdBf}\left(\tilde{T}^{\alpha}, \tilde{F}^{\alpha}\right)$ 
has an extension in $\mathcal{C}$. A Boolean function $f \in \mathcal{E}\left(\tilde{T}^{\alpha}, \tilde{F}^{\alpha}\right) \cap \mathcal{C}$ will be called a consistent extension of $(\tilde{T}, \tilde{F})$ in the class $\mathcal{C}$.

\section{Problem CE $(\mathcal{C})$}

Input: $\quad \mathrm{A} \operatorname{pBmb}(\tilde{T}, \tilde{F})$, where $\tilde{T}, \tilde{F} \subseteq \mathbb{M}^{n}$.

Question: Does $(\tilde{T}, \tilde{F})$ have a consistent extension in class $\mathcal{C}$ ?

Let us note that, in case $(\tilde{T}, \tilde{F})$ has a consistent extension, the output of $\mathrm{CE}(\mathcal{C})$ might not be unique, and at an important extreme end, it may occur that for every possible interpretations of the missing bits the obtained pdBf has an extension belonging to $\mathcal{C}$. Let us call a $\mathrm{pBmb}(\tilde{T}, \tilde{F})$ fully consistent with the class $\mathcal{C}$ if this occurs, i.e. if $\left(\tilde{T}^{\alpha}, \tilde{F}^{\alpha}\right)$ has an extension in $\mathcal{C}$ for every $\alpha \in \mathbb{B}^{S}$ (the corresponding extensions may differ for different $\alpha$ 's.)

\section{Problem $\mathrm{FC}(\mathcal{C})$}

Input: $\quad \mathrm{A} \operatorname{pBmb}(\tilde{T}, \tilde{F})$, where $\tilde{T}, \tilde{F} \subseteq \mathbb{M}^{n}$.

Question: Is $(\tilde{T}, \tilde{F})$ fully consistent with the class $\mathcal{C}$ ?

Let us remark that, unlike for problem $\mathrm{CE}(\mathcal{C})$, confirming a YES for problem $\mathrm{FC}(\mathcal{C})$ might become a computational burden because one may have to provide $2^{|S|}$ different extensions, for each possible assignment to the missing bits of $(\tilde{T}, \tilde{F})$, even if each extension $f \in \mathcal{E}\left(\tilde{T}^{\alpha}, \tilde{F}^{\alpha}\right) \cap \mathcal{C}$ has a small representation. For this reason, we shall consider a special case in which in fact all these extensions coincide. Let us call a Boolean function $f$ a robust extension of a given $\mathrm{pBmb}$ $(\tilde{T}, \tilde{F})$ if

$$
f\left(a^{\alpha}\right)=1 \text { and } f\left(b^{\alpha}\right)=0 \text { for all } a \in \tilde{T}, b \in \tilde{F} \text { and for all } \alpha \in \mathbb{B}^{S} .
$$

The corresponding decision problem can be stated as follows.

\section{Problem RE $(\mathcal{C})$}

Input: $\quad \mathrm{A} \operatorname{pBmb}(\tilde{T}, \tilde{F})$, where $\tilde{T}, \tilde{F} \subseteq \mathbb{M}^{n}$.

Question: Does $(\tilde{T}, \tilde{F})$ have a robust extension in class $\mathcal{C}$ ?

Let us denote by $\mathcal{E}(\tilde{T}, \tilde{F})$ the family of all robust extensions of a given $\mathrm{pBmb}$ $(\tilde{T}, \tilde{F})$.

Let us remark now that even in this special case, the computational verification of a YES may not be an easy problem. Consider, for instance, the case when the output function $f$ is represented by a DNF. Then, verifying that $f\left(a^{\alpha}\right)=1$ holds for a vector $a \in \tilde{T}$ and for all $\alpha \in \mathbb{B}^{S(a)}$ might be as difficult as the tautology problem, which is known to be co-NP-complete even if its input is restricted to 3 -DNF-s (see [12]) 1]

\footnotetext{
${ }^{1}$ The tautology problem is to decide if a given $\operatorname{DNF} \varphi$ satisfies $\varphi \equiv 1$. This is the complement of satisfiability problem, which is to decide, given a $\mathrm{CNF} \varphi$, if there exists a vector $v$ for which $\varphi(v)=1$.
} 
We shall therefore consider a further special case, when such difficulties will not arise. Consider an elementary conjunction (i.e., term)

$$
t(\mathbf{x})=\bigwedge_{j \in P} x_{j} \bigwedge_{j \in N} \bar{x}_{j}
$$

for some subsets $P, N \subseteq\{1,2, \ldots, n\}$ with $P \cap N=\emptyset$. We shall call $t$ a robust term with respect to a $\mathrm{pBmb}(\tilde{T}, \tilde{F})$ and a vector $a \in \tilde{T}$, if $t\left(a^{\alpha}\right)=1$ for all $\alpha \in \mathbb{B}^{S(a)}$ and $t\left(b^{\beta}\right)=0$ for all $b \in \tilde{F}$ and $\beta \in \mathbb{B}^{S(b)}$. Let us note that a term $t$ is robust with respect to $(\tilde{T}, \tilde{F})$ and $a \in \tilde{T}$ if and only if $S(a) \cap(P \cup N)=\emptyset$, and $(O N(b) \cap N) \cup(O F F(b) \cap P) \neq \emptyset$ for all $b \in \tilde{F}$, conditions which are all easy to check. Let us then call a Boolean function $f$ a very robust extension of $(\tilde{T}, \tilde{F})$, if it is a robust extension which can be represented by a disjunction of robust terms.

\section{$\operatorname{Problem} \operatorname{VR}(\mathcal{C})$}

Input: $\quad \mathrm{A} \operatorname{pBmb}(\tilde{T}, \tilde{F})$, where $\tilde{T}, \tilde{F} \subseteq \mathbb{M}^{n}$.

Question: Does $(\tilde{T}, \tilde{F})$ have a very robust extension in class $\mathcal{C}$ ?

provided.

Let us denote by $\mathcal{E}^{*}(\tilde{T}, \tilde{F})$ the family of all very robust extensions of the $\mathrm{pBmb}$ $(\tilde{T}, \tilde{F})$.

Problems $\mathrm{CE}(\mathcal{C})$ and $\operatorname{RE}(\mathcal{C})$ and some related optimization problems have been considered extensively for various classes in [7!9]. In this paper we concentrate on the relations between $\operatorname{FC}(\mathcal{C}), \operatorname{RE}(\mathcal{C})$ and $\operatorname{VR}(\mathcal{C})$. It is quite immediate to see from the above definitions that very robust extensions are robust as well, and that pBmbs which have robust extensions in a given class $\mathcal{C}$ are also fully consistent with that class.

Somewhat surprisingly, we can show that for many very general families of classes $\mathcal{C}$, these three problems $\operatorname{FC}(\mathcal{C}), \operatorname{RE}(\mathcal{C})$ and $\operatorname{VR}(\mathcal{C})$ are equivalent. However such an equivalence does not hold for all classes. In fact, for certain classes $\mathcal{C}$, problem $\operatorname{RE}(\mathcal{C})$ is polynomially solvable, while $\mathrm{FC}(\mathcal{C})$ is co-NP-complete.

\section{Classes of Boolean Functions}

We shall assume in the sequel that Boolean functions (functions, in short) are represented either by an explicit algebraic form, or by an oracle. In either case, it is possible to compute the values of such a function for given input vectors. In each case in the sequel, we shall make clear what is the representation of the considered family of functions.

Let us call an elementary conjunction of literals a term. The most common representation we shall consider for a function $f$ will be either a disjunctive normal form (or DNF in short), which is a disjunction of terms.

For two functions $f$ and $g$, we shall write $f \leq g$, if $f(x)=1$ always implies $g(x)=1$, and $f<g$ if $f \leq g$ and $f \neq g$. For a Boolean expression $A$, 
let us denote by $\bar{A}=1-A$ its negation. The components of the (unknown) vector $x=\left(x_{1}, \ldots, x_{n}\right)$ will be called Boolean variables, while variables and their complements together are called literals.

A term $t$ is called an implicant of a function $f$ if $t \leq f$, and it is a prime implicant if $t$ is a maximal implicant, i.e., $t \leq f$ and no term $t^{\prime}$ exists such that $t<t^{\prime} \leq f$. It is well-known that every Boolean function can be represented by the DNF formed by the disjunction of all of its prime implicants. It is also well-known that in general, there are many other DNFs representing the same function.

We shall consider many different classes of Boolean functions, whose definitions will be either via some representation independent functional properties, or by properties of some of the DNF representations, or via some other representations.

A large family of classes of the first type are the transitive or generalized monotone classes. Let us consider a partial order $\preceq$ of the vectors $\mathbb{B}^{n}$, and let us say that a function $f$ is $\preceq$-monotone, if $f(x) \leq f(y)$ holds whenever $x \preceq y$. For a given partial order $\preceq$ on $\mathbb{B}^{n}$, let us denote by $\mathcal{C} \preceq$ the family of all $\preceq$-monotone functions. Then, we shall call a class $\mathcal{C}$ transitive, if there exists a partial order

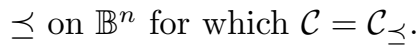

Most notable examples for transitive classes are the family of positive (also called monotone) functions, $\mathcal{C}_{+}=\mathcal{C}_{\geq}$, where $f \in \mathcal{C}_{+}$if $f(x) \geq f(y)$ holds whenever $x \geq y$ holds (componentwise), and the family $\mathcal{C}_{\text {reg }}$ of regular Boolean functions, where $\mathcal{C}_{\text {reg }}=\mathcal{C}_{\succ}$ for the relation $\succ$, defined by $x \succ y$ if and only if $\sum_{j=1}^{k} x_{j} \geq \sum_{j=1}^{k} y_{j}$ for all $1 \leq k \leq n$.

Another frequently used partial order on the Boolean cube is a "tilted" monotone order. To an arbitrary vector $b \in \mathbb{B}^{n}$, we can associate a partial order $\geq_{b}$ of the Boolean cube $\mathbb{B}^{n}$ by defining that $v \geq_{b} w$ holds if and only if $v \oplus b \geq w \oplus b$ holds, where $\oplus$ denotes the exclusive-or operation (the componentwise $\bmod 2$ addition, e.g. $(1100) \oplus(0110)=(1010))$. In other words, $\geq_{b}$ is like the regular monotone order $\geq$ in which $b$ plays the role of the zero-vector $(0,0, \ldots, 0)$, and $\bar{b}$ is the maximum vector. The family of $\geq_{b}$-monotone functions will be denoted by $\mathcal{C}_{\geq_{b}}$. Thus, in particular $\mathcal{C}_{+}=\mathcal{C}_{\geq_{0}}$ holds. Let us finally remark that the family $\mathcal{C}_{\text {all }}$ of all Boolean functions itself is a transitive class, corresponding to the "empty" partial order on $\mathbb{B}^{n}$.

Some other non-transitive classes, defined via a representation independent property can be obtained by taking the union of various transitive classes. For instance, a function $f$ is called unate if it is $\geq_{b}$-monotone for some vector $b \in \mathbb{B}^{n}$. The family of unate functions, hence is the union of all the $\geq_{b}$-monotone classes, $\mathcal{C}_{\text {unate }}=\bigcup_{b \in \mathbb{B}^{n}} \mathcal{C}_{\geq_{b}}$.

Other examples for classes defined via a representation independent property include the family of self-dual functions $\mathcal{C}_{S D}$, consisting of functions $f$ for which $f=f^{d}$, where the dual $f^{d}$ of a Boolean function $f$ is defined by $f\left(x_{1}, \ldots, x_{n}\right)=$ $\bar{f}\left(\bar{x}_{1}, \ldots, \bar{x}_{n}\right)$. Similarly, the family of dual-minor functions $\mathcal{C}_{D \text {-minor }}$ consists of the functions satisfying the inequality $f \leq f^{d}$, while the class of dual-major functions $\mathcal{C}_{D \text {-major }}$ is formed by the functions satisfying $f \geq f^{d}$. 
Another large family of classes, the so called DNF-classes are defined via their DNF representation. Let us consider a family of terms $\mathbb{T}$, and let us define the corresponding DNF-class $\mathcal{C}_{\mathbb{T}}$ by $\mathcal{C}_{\mathbb{T}}=\left\{f(x)=\bigvee_{t \in \mathcal{S}} t(x) \mid \mathcal{S} \subseteq \mathbb{T}\right\}$ as the collection of all Boolean functions formed by the disjunction of a subset of terms from $\mathbb{T}$. For example, if $\mathbb{T}$ consists of all terms of degree at most $k$ (elementary conjunctions involving at most $k$ literals), then the corresponding DNF-class is the family of the so-called $k$-DNFs, $\mathcal{C}_{k-D N F}$. The special cases of linear functions $\left(\mathcal{C}_{1-D N F}\right)$ and quadratic functions $\left(\mathcal{C}_{2-D N F}\right)$ should, in particular be mentioned. Another notable example for a DNF-class is the family of Horn functions, $\mathcal{C}_{H o r n}$. A Boolean function $f$ is called Horn, if it can be represented by a DNF in which every term involves at most one negative variable. In other words, if $\mathbb{T}$ is the family of terms involving at most one negative variable, then Horn functions form the corresponding DNF-class, $\mathcal{C}_{\mathbb{T}}=\mathcal{C}_{\text {Horn }}$.

Let us remark that DNF-classes $\mathcal{C}_{\mathbb{T}}$ for which $\mathbb{T}$ is closed under consensus (for definition see Section 51) will play a special role due to the property that, for such a DNF, all its prime implicants must also belong to $\mathbb{T}$. Among consensus closed classes we should mention 2-DNFs, Horn functions, and $\geq_{b}$-monotone functions.

Given the Boolean functions $f$ and $g$, we shall call $g$ a minor of $f$, and will denote it by $g \sqsubseteq f$, if $g$ can be represented by a disjunction of some of the prime implicants of $f$. Let us then call a class $\mathcal{C}$ minor closed if $f \in \mathcal{C}$ and $g \sqsubseteq f$ imply $g \in \mathcal{C}$. Minor closed classes include, in particular, all consensus closed DNF classes, and unions of those, such as renamable Horn functions, unate functions, q-Horn functions (see e.g., [3]), etc.

One other important class of functions, the class $\mathcal{C}_{T H}$ of threshold functions, is defined usually by a different representation. A Boolean function $f$ is called threshold if there exist real numbers $w_{1}, \ldots, w_{n}$ and $w_{0}$ such that $f(x)=1$ if and only if the inequality $\sum_{j=1}^{n} w_{j} x_{j} \geq w_{0}$ holds. In other words, $f$ is threshold exactly when the sets $T(f)$ and $F(f)$, viewed as point sets in the Euclidean space $\mathbb{R}^{n}$, are linearly separable. Of course, threshold functions could also be represented by DNFs (or CNFs), but for most threshold functions such a representation would be much less efficient computationally.

\section{Equivalencies between $\operatorname{RE}(\mathcal{C})$ and $\mathrm{FC}(\mathcal{C})$}

In this section we shall show a series of results claiming, somewhat surprisingly, the equivalence of problems $\operatorname{RE}(\mathcal{C})$ and $\mathrm{FC}(\mathcal{C})$, under some widely applicable conditions. Let us remark here that two decision problems are equivalent if they have the same output (YES or NO) for all possible input. Equivalent decision problems are of course also equivalent computationally.

Let us also note that due to the space limitations we could not include all the proofs here, and we refer the reader to [10] for the missing details.

Let us first consider those classes $\mathcal{C}$ of Boolean functions which are closed under conjunction and disjunction; i.e., $f \wedge g \in \mathcal{C}$ and $f \vee g \in \mathcal{C}$ for all $f, g \in \mathcal{C}$.

Theorem 1. Let us assume that the class $\mathcal{C}$ of Boolean functions is closed under conjunction and disjunction. Then problems $\mathrm{RE}(\mathcal{C})$ and $\mathrm{FC}(\mathcal{C})$ are equivalent. 
Proof. Since the existence of a robust extension always implies full consistency, we only show the opposite implication. Assuming that the $\mathrm{pBmb}(\tilde{T}, \tilde{F})$ is fully consistent with $\mathcal{C}$, we show that $(\tilde{T}, \tilde{F})$ has also a robust extension.

By the assumption, for every pair $\alpha \in \mathbb{B}^{S(\tilde{T})}$ and $\beta \in \mathbb{B}^{S(\tilde{F})}$, there exists an extension $f_{\alpha, \beta} \in \mathcal{C} \cap \mathcal{E}\left(\tilde{T}^{\alpha}, \tilde{F}^{\beta}\right)$. Let us then consider the Boolean function $f$ defined by

$$
f=\bigvee_{\alpha \in \mathbb{B}^{S(\tilde{T})}}\left(\bigwedge_{\beta \in \mathbb{B}^{S(\tilde{F})}} f_{\alpha, \beta}\right) .
$$

We claim that $f$ is a robust extension of $(\tilde{T}, \tilde{F})$ in the class $\mathcal{C}$. First, $f \in \mathcal{C}$ follows from our assumption that $\mathcal{C}$ is closed under conjunction and disjunction.

To see that $f$ is a robust extension of $(\tilde{T}, \tilde{F})$, let us first consider a vector $a \in \tilde{T}$ and an arbitrary assignment $\alpha^{*} \in \mathbb{B}^{S(\tilde{T})}$. Since $a^{\alpha^{*}} \in \tilde{T}^{\alpha^{*}}$, we have $\bigwedge_{\beta \in \mathbb{B}^{S(\tilde{F})}} f_{\alpha^{*}, \beta}\left(a^{\alpha^{*}}\right)=1$ by the fact that $f_{\alpha^{*}, \beta} \in \mathcal{E}\left(\tilde{T}^{\alpha^{*}}, \tilde{F}^{\beta}\right)$ for all $\beta \in \mathbb{B}^{S(\tilde{F})}$. Thus $f\left(a^{\alpha^{*}}\right)=1$ is implied, for all $\alpha^{*} \in \mathbb{B}^{S(\tilde{T})}$.

Analogously, for a vector $b \in \tilde{F}$ and an assignment $\beta^{*} \in \mathbb{B}^{S(\tilde{F})}$ we can observe first that $f_{\alpha, \beta^{*}}\left(b^{\beta^{*}}\right)=0$ holds for all $\alpha \in \mathbb{B}^{S(\tilde{T})}$, implied again by $f_{\alpha, \beta^{*}} \in \mathcal{E}\left(\tilde{T}^{\alpha}, \tilde{F}^{\beta^{*}}\right)$. Thus, in this case $\bigwedge_{\beta \in \mathbb{B}^{S(\tilde{F})}} f_{\alpha, \beta}\left(b^{\beta^{*}}\right)=0$ follows for all $\alpha \in \mathbb{B}^{S(\tilde{T})}$, implying hence $f\left(b^{\beta^{*}}\right)=0$, for all $\beta^{*} \in \mathbb{B}^{S(\tilde{F})}$.

These two observations then show that $f$ is indeed a robust extension of $(\tilde{T}, \tilde{F})$.

It is easy to see that transitive classes are closed under conjunction and disjunction (and even more, a class is transitive if and only if it is closed under conjunction and disjunction, see [2]) and hence the following corollary is implied by the above theorem:

Corollary 1. Problems $\mathrm{RE}(\mathcal{C})$ and $\mathrm{FC}(\mathcal{C})$ are equivalent for all transitive classes, including $\mathcal{C}_{\text {all }}, \mathcal{C}_{+}, \mathcal{C}_{\text {regular }}$ and $\mathcal{C}_{\geq_{b}}$ for all $b \in \mathbb{B}^{n}$.

Let us consider next certain lattice like transitive relations. We shall say that a partial order $\succeq$ on $\mathbb{B}^{n}$ is cube-lattice like if there is a unique $\succeq$-maximum and a unique $\succeq$-minimum in any subcube of $\mathbb{B}^{n}$, or equivalently, if for every term $t$, there are unique vectors $u, v \in T(t)$ such that $u \succeq w \succeq v$ holds for all $w \in T(t)$. Let us note that all partial orders mentioned in the previous section (e.g., $\geq_{b}$ for $b \in \mathbb{B}^{n}, \succ$, etc.) are cube-lattice like, and there are many others. For instance, an arbitrary permutation of the $2^{n}$ vertices of $\mathbb{B}^{n}$, viewed as a linear order, is cube-lattice like.

For vectors $v, w \in \mathbb{M}^{n}$, we write $v \approx w$ if there is an assignment $\alpha \in \mathbb{B}^{S(\{v, w\})}$ such that $v^{\alpha}=w^{\alpha}$, and we say that $v$ is potentially identical with $w$. For example, if $v=(1,0, *, 1, *)$ and $w=(1, *, 0,1, *)$ then $v \approx w$ holds.

To every vector $a \in \mathbb{M}^{n}$ we can associate the subcube $B(a)=\left\{u \in \mathbb{B}^{n} \mid u \approx\right.$ $a\}=\left\{a^{\alpha} \mid \alpha \in \mathbb{B}^{S(a)}\right\}$ of $\mathbb{B}^{n}$ consisting of all Boolean vectors one can obtain from $a$ by assigning binary values to its missing bits. Given a vector $a \in \mathbb{M}^{n}$ and a cube-lattice like partial order $\succeq$ on $\mathbb{B}^{n}$, let us denote by $a^{+} \in B(a)$ (resp., 
$a^{-} \in B(a)$ ) the unique $\succeq$-maximal vector (resp., the unique $\succeq$-minimal vector) in the subcube $B(a)$. Furthermore, for any subset $S \subseteq \mathbb{M}^{n}$ let $S^{+}=\left\{a^{+} \mid a \in S\right\}$ and $S^{-}=\left\{a^{-} \mid a \in S\right\}$ denote the corresponding subsets of Boolean vectors.

With these notations, we can state the following generalization of [9, Lemma $1]$ :

Lemma 1. If $\mathcal{C}$ is an arbitrary subfamily of a transitive class $\mathcal{C}_{\succeq}$ with a cubelattice like partial order $\succeq$, then a $p B m b(\tilde{T}, \tilde{F})$ has a robust extension in $\mathcal{C}$ if and only if the $\operatorname{pdBf}\left(\tilde{T}^{-}, \tilde{F}^{+}\right)$has an extension in $\mathcal{C}$.

Proof. Since $\tilde{T}^{-}=\tilde{T}^{\alpha^{*}}$ for some $\alpha^{*} \in \mathbb{B}^{S(\tilde{T})}$, and $\tilde{F}^{+}=\tilde{F}^{\beta^{*}}$ for some $\beta^{*} \in$ $\mathbb{B}^{S(\tilde{F})}$, it follows that any robust extension of $(\tilde{T}, \tilde{F})$ will be an extension of $\left(\tilde{T}^{-}, \tilde{F}^{+}\right)$, by the definition of a robust extension.

To see the reverse direction, let us assume that $f \in \mathcal{E}\left(\tilde{T}^{-}, \tilde{F}^{+}\right) \cap \mathcal{C}$. Since all functions in $\mathcal{C}$ are $\succeq$-monotone, and since $a^{\alpha} \succeq a^{-}$holds, we have $f\left(a^{\alpha}\right) \geq$ $f\left(a^{-}\right)=1$ implied for all $a \in \tilde{T}$ and $\alpha \in \mathbb{B}^{S(\tilde{T})}$. Similarly, $b^{\beta} \leq b^{+}$and $f\left(b^{+}\right)=0$ implies $f\left(b^{\beta}\right) \leq f\left(b^{+}\right)=0$ for all $b \in \tilde{F}$ and $\beta \in \mathbb{B}^{S(\tilde{F})}$. Hence, this function $f$ is also a robust extension of $(\tilde{T}, \tilde{F})$ in $\mathcal{C}$.

This lemma immediately implies the following statement.

Theorem 2. If the class $\mathcal{C}$ is a subfamily of a transitive class $\mathcal{C}_{\succeq}$ with a cubelattice like partial order $\succeq$, then problems $\operatorname{RE}(\mathcal{C})$ and $\mathrm{FC}(\mathcal{C})$ are equivalent.

Proof. Indeed, if the $\operatorname{pBmb}(\tilde{T}, \tilde{F})$ is fully consistent with the class $\mathcal{C}$, then the $\operatorname{pdBf}\left(\tilde{T}^{-}, \tilde{F}^{+}\right)$has an extension $f \in \mathcal{C} \cap \mathcal{E}\left(\tilde{T}^{-}, \tilde{F}^{+}\right)$. This $f$ will then be a robust extension of $(\tilde{T}, \tilde{F})$ in $\mathcal{C}$ by Lemma 1 . The converse direction is obvious by the definitions.

Corollary 2. For any subfamily $\mathcal{C}$ of $\mathcal{C}_{+}, \mathcal{C}_{\text {regular }}$ and $\mathcal{C}_{\geq_{b}}$ for any $b \in \mathbb{B}^{n}$, problems $\operatorname{RE}(\mathcal{C})$ and $\mathrm{FC}(\mathcal{C})$ are equivalent.

Let us next consider DNF-classes.

Theorem 3. Problems $\operatorname{RE}(\mathcal{C})$ and $\mathrm{FC}(\mathcal{C})$ are equivalent for all DNF-classes $\mathcal{C}=\mathcal{C}_{\mathbb{T}}$.

Proof. Let us only show that fully consistency implies the existence of a robust extension, since the converse direction is immediate from the definition.

Observe first that, given a true vector $a \in \tilde{T}$ and an assignment $\alpha \in \mathbb{B}^{S(a)}$, each false vector $b \in \tilde{F}$ has a unique assignment $\beta=\beta(\alpha) \in \mathbb{B}^{S(b)}$ minimizing the Hamming distance between the Boolean vectors $a^{\alpha}$ and $b^{\beta}$.

Let us fix an arbitrary vector $a \in \tilde{T}$ and an assignment $\alpha \in \mathbb{B}^{S(\tilde{T})}$, and define $\beta^{*} \in \mathbb{B}^{S(\tilde{F})}$ as the unique assignment which coincides with $\beta(\alpha) \in \mathbb{B}^{S(b)}$ for all $b \in \tilde{F}$. Such an assignment obviously can be constructed by concatenating the $\beta(\alpha)$ assignments for $b \in \tilde{F}$, since the sets $S(b)$ for $b \in \tilde{F}$ are pairwise disjoint.

Since $(\tilde{T}, \tilde{F})$ is fully consistent with $\mathcal{C}_{\mathbb{T}}$ by our assumption, there exists a Boolean function $g \in \mathcal{C}_{\mathbb{T}} \cap \mathcal{E}\left(\tilde{T}^{\alpha}, \tilde{F}^{\beta^{*}}\right)$. Since $a^{\alpha}$ is a true vector of such an extension, $g$ must have a term $t_{a, \alpha} \in \mathbb{T}$ for which $t_{a, \alpha}\left(a^{\alpha}\right)=1$. 
We claim that $t_{a, \alpha}\left(b^{\beta}\right)=0$ holds for all $b \in \tilde{F}$ and $\beta \in \mathbb{B}^{S(\tilde{F})}$. To see this, let us observe that for every vector $b \in \tilde{F}$ there must be a literal in $t_{a, \alpha}$ at which $b^{\beta^{*}}$ and $a^{\alpha}$ are different, since otherwise $t_{a, \alpha}\left(b^{\beta^{*}}\right)=t_{a, \alpha}\left(a^{\alpha}\right)=1$ would follow, contradicting the fact that $g$ is an extension of the $\operatorname{pdBf}\left(\tilde{T}^{\alpha^{*}}, \tilde{F}^{\beta^{*}}\right)$. Then this literal does not correspond to any component of $S(b)$, otherwise we could switch its value in $\beta^{*}$ to decrease the Hamming distance to $a^{\alpha}$. Thus, this literal does not agree with any $b^{\beta}$ for $\beta \in \mathbb{B}^{S(\tilde{F})}$, and hence the claim follows.

Therefore, the Boolean function defined by

$$
f=\bigvee_{a \in \tilde{T}, \alpha \in \mathbb{B}^{S(\tilde{T})}} t_{a, \alpha} .
$$

is a robust extension of $(\tilde{T}, \tilde{F})$ in $\mathcal{C}_{\mathbb{T}}$. Indeed, the equations $f\left(b^{\beta}\right)=0$ hold for all $b \in \tilde{F}$ and $\beta \in \mathbb{B}^{S(\tilde{F})}$ according to the above claim. Furthermore, for a true vector $a \in \tilde{T}$ and an arbitrary assignment $\alpha \in \mathbb{B}^{S(\tilde{T})}$ we have $f\left(a^{\alpha}\right)=1$ implied by $t_{a, \alpha}\left(a^{\alpha}\right)=1$.

Corollary 3. Problems $\operatorname{RE}(\mathcal{C})$ and $\mathrm{FC}(\mathcal{C})$ are equivalent for $\mathcal{C}_{\text {all }}, \mathcal{C}_{+}, \mathcal{C}_{k-D N F}$, $\mathcal{C}_{\text {Horn }}$.

Let us finally consider self-dual, dual-minor and dual-major functions.

Theorem 4. Problems $\operatorname{RE}(\mathcal{C})$ and $\mathrm{FC}(\mathcal{C})$ are equivalent for $\mathcal{C}=\mathcal{C}_{S D}$ (resp., $\mathcal{C}_{D \text {-minor }}$ and $\left.\mathcal{C}_{D \text {-major }}\right)$, if $(*, *, \ldots, *) \notin \tilde{T} \cup \tilde{F}($ resp., $(*, *, \ldots, *) \notin \tilde{T}$ and $(*, *, \ldots, *) \notin \tilde{F})$.

Corollaries 1, 2, 3] and Theorem 4, together with the complexity results of $\mathrm{RE}(\mathcal{C})$ in 9], imply the following theorem.

Theorem 5. Problem $\mathrm{FC}(\mathcal{C})$ is polynomially decidable for $\mathcal{C}=\mathcal{C}_{\text {all }}, \mathcal{C}_{+}, \mathcal{C}_{\text {regular }}$ $\mathcal{C}_{k-D N F}^{+}($for a constant $k), \mathcal{C}_{k-D N F}($ for $k=1,2), \mathcal{C}_{H o r n}, \mathcal{C}_{S D}^{(+)}, \mathcal{C}_{D \text {-minor }}^{(+)}$and $\mathcal{C}_{D-\text { major }}^{(+)}$, where $\mathcal{C}_{X}^{+}$denotes the class of positive functions in $\mathcal{C}_{X}$, while it is co-NP-complete for $\mathcal{C}=\mathcal{C}_{k-D N F}($ for a constant $k \geq 3)$.

\section{$5 \quad$ Very Robust Extensions}

Very robust extensions play a computationally important role, since when they exists, they usually can efficiently be constructed. For instance, we shall show below that for most DNF-classes $\mathcal{C}$, if $\operatorname{RE}(\mathcal{C})$ can be solved in polynomial time, then a very robust extension can also be provided at the same time.

Let us recall that a term

$$
t\left(x_{1}, \ldots, x_{n}\right)=\bigwedge_{j \in P} x_{j} \bigwedge_{j \in N} \bar{x}_{j}
$$


is called a robust term with respect to $a \in \tilde{T}$ for a $\mathrm{pBmb}(\tilde{T}, \tilde{F})$, if $t\left(a^{\alpha}\right)=1$ for all $\alpha \in \mathbb{B}^{S(a)}$, and $t\left(b^{\beta}\right)=0$ for all $b \in \tilde{F}$ and $\beta \in \mathbb{B}^{S(b)}$. In other words, if and only if

$$
\begin{gathered}
P \subseteq O N(a) \text { and } N \subseteq O F F(a), \text { and } \\
P \cap O F F(b) \neq \emptyset \text { or } N \cap O N(b) \neq \emptyset \text { textforeveryvector } b \in \tilde{F} .
\end{gathered}
$$

Since both of these conditions are independent of the assignments to missing bits of $(\tilde{T}, \tilde{F})$, checking these conditions is quite straightforward. Therefore, verifying that a given DNF is a very robust extension of a $\mathrm{pBmb}(\tilde{T}, \tilde{F})$ can be done in linear time in the size of $(\tilde{T}, \tilde{F})$. It is also clear from the definition that in a very robust extension one never needs more than $|\tilde{T}|$ terms.

Furthermore, looking at conditions (1) and (2), it is easy to see that finding a robust term for a given $\mathrm{pBmb}(\tilde{T}, \tilde{F})$ and vector $a \in \tilde{T}$ reduces to a feasibility question in an associated setcovering problem, and hence it is computationally tractable in most cases. The above immediately imply for instance the following statement.

Theorem 6. Problem $\operatorname{VR}(\mathcal{C})$ can be solved in polynomial time for $\mathcal{C}=\mathcal{C}_{\text {all }}$, for $\mathcal{C}_{\geq_{b}}$ for with $b \in \mathbb{B}^{n}$ (thus in particular for $\mathcal{C}=\mathcal{C}_{+}$), for $\mathcal{C}=\mathcal{C}_{H o r n}$ (and for all related classes, such as $k$-quasi Horn and $k$-quasi reverse Horn for any fixed $k$ ), and for $\mathcal{C}=\mathcal{C}_{k-D N F}$ with $k$ fixed.

Let us recall that a class $\mathcal{C}$ is minor closed, if $f \in \mathcal{C}$ and $g \sqsubseteq f$ imply $g \in \mathcal{C}$. To discuss properties of very robust extensions, we shall further recall the consensus method and some of its properties (see e.g., [1415]). Given two terms $t=\bigwedge_{j \in P} x_{j} \bigwedge_{j \in N} \bar{x}_{j}$ and $t^{\prime}=\bigwedge_{j \in P^{\prime}} x_{j} \bigwedge_{j \in N^{\prime}} \bar{x}_{j}$, we say that they are in conflict at variable $x_{j}$ if $j \in\left(P \cap N^{\prime}\right) \cup\left(N \cap P^{\prime}\right)$ (i.e., if $x_{j}$ appears in one and $\bar{x}_{j}$ appears in the other). If $t$ and $t^{\prime}$ are in conflict at exactly one of the variables, then their consensus is a term $t^{\prime \prime}=\left[t, t^{\prime}\right]$ defined by

$$
t^{\prime \prime}=\bigwedge_{j \in\left(P \backslash N^{\prime}\right) \cup\left(P^{\prime} \backslash N\right)} x_{j} \bigwedge_{j \in\left(N \backslash P^{\prime}\right) \cup\left(N^{\prime} \backslash P\right)} \bar{x}_{j} .
$$

In other words, the consensus of $t$ and $t^{\prime}$ is the conjunction of all the literals appearing in these terms, except the two, corresponding to the conflicting variable. It is easy to see that the inequality $t^{\prime \prime} \leq t \vee t^{\prime}$ holds, and that $t^{\prime \prime}$ is maximal for this property. This implies, in particular that if $t$ and $t^{\prime}$ are implicants of the Boolean function $f$, then their consensus $t^{\prime \prime}=\left[t, t^{\prime}\right]$ (when exists) is also an implicant of $f$. The consensus method is the algorithm, in which consensuses of implicants of a given DNF of $f$ are formed as long as new implicants are generated. It is well-known (see e.g., [14]) that this method is complete in the sense that all prime implicants of $f$ will be obtained in this way, starting from any DNF representation of $f$. Of course, all these notions and results can straightforwardly be translated for CNF representations using De Morgan's laws. The corresponding operation between clauses is known as resolution. 
Returning to robust terms, we are now ready to prove the following statement.

Lemma 2. If $f$ is a robust extension of the $p B m b(\tilde{T}, \tilde{F})$, then for every vector $a \in \tilde{T}, f$ has a prime implicant $t_{a} \leq f$, which is a robust term with respect to $a$.

Proof. Let us consider an (arbitrary) DNF representation of $f$ :

$$
f=\bigvee_{i=1}^{m} t_{i}
$$

where

$$
t_{i}=\bigwedge_{j \in P_{i}} x_{j} \bigwedge_{j \in N_{i}} \bar{x}_{j}
$$

Given a vector $a \in \tilde{T}$, we substitute $x_{j}=1$ for variables with $j \in O N(a)$, and $x_{j}=0$ for variables with $j \in O F F(a)$ into (3). Let $I \subseteq\{1,2, \ldots, m\}$ denote the set of indices of those terms of (3) which do not vanish after this substitution. For terms $t_{i}$ for $i \in I$, we have

$$
P_{i} \cap O F F(a)=\emptyset \text { and } N_{i} \cap O N(a)=\emptyset .
$$

Let us denote the resulting DNF by $f^{\prime}=\bigvee_{i \in I} t_{i}^{\prime}$, where

$$
t_{i}^{\prime}=\bigwedge_{j \in P_{i} \backslash O N(a)} x_{j} \bigwedge_{j \in N_{i} \backslash O F F(a)} \bar{x}_{j} .
$$

Since $1=f\left(a^{\alpha}\right)=f^{\prime}\left(a^{\alpha}\right)$ holds for all $\alpha \in \mathbb{B}^{S(a)}$, it follows that $f^{\prime}$ is the constant 1 function, and thus the only prime implicant 1 of $f^{\prime}$ can be obtained by a chain of consensuses, starting with the terms of $f^{\prime}$. Let us note that if the terms $t_{i}^{\prime}$ and $t_{k}^{\prime}$ for some $i \neq k, i, k \in I$ have a consensus, then so do the terms $t_{i}$ and $t_{k}$. Furthermore, the variables $x_{j}, j \in O N(a)$, appear only positively, and the variables $x_{j}, j \in O F F(a)$, appear only negatively in the resulting consensus $\left[t_{i}, t_{k}\right]$. Applying this observation recursively, we can repeat the same chain of consensuses which produced 1 from $t_{i}^{\prime}, i \in I$, with the corresponding terms $t_{i}$, $i \in I$, yielding an implicant $t_{a}^{\prime}$ of $f$.

Clearly, $t_{a}^{\prime}$ involves only literals $x_{j}$ for some $j \in O N(a)$ and $\bar{x}_{j}$ for some $j \in O F F(a)$, and thus $t_{a}^{\prime}$ satisfies condition (1). Therefore, by deleting some literals from $t_{a}^{\prime}$ if needed, we can obtain a prime implicant $t_{a}$ of $f$ still satisfying (1).

Let us note finally that any (prime) implicant of $f$ must satisfy conditions (2) simply because $f$ is a robust extension of $(\tilde{T}, \tilde{F})$.

Theorem 7. If $\mathcal{C}$ is a minor closed class, then problems $\operatorname{RE}(\mathcal{C})$ and $\operatorname{VR}(\mathcal{C})$ are equivalent. 
Proof. Since a very robust extension is also a robust extension, let us prove only the non-trivial direction of the stated equivalence.

Let us assume that $(\tilde{T}, \tilde{F})$ is a pBmb which has a robust extension $f \in$ $\mathcal{E}(\tilde{T}, \tilde{F}) \cap \mathcal{C}$. According to Lemma 2, for every $a \in \tilde{T}$, $f$ has a prime implicant $t_{a} \leq f$ which is a robust term of $(\tilde{T}, \tilde{F})$. We claim that the Boolean function

$$
g=\bigvee_{a \in \tilde{T}} t_{a}
$$

is a very robust extension of $(\tilde{T}, \tilde{F})$ in the class $\mathcal{C}$.

Clearly, $g \leq f$ is a minor of $f$ by its definition, hence $g \in \mathcal{C}$ is implied by the facts that $\mathcal{C}$ is minor closed and $f \in \mathcal{C}$. Since $f \in \mathcal{E}(\tilde{T}, \tilde{F})$, the inequality $g \leq f$ also implies that $g\left(b^{\beta}\right) \leq f\left(b^{\beta}\right)=0$ for all $b \in \tilde{F}$ and $\beta \in \mathbb{B}^{S(b)}$. Also, since $g$ contains a robust term $t_{a}$ for every $a \in \tilde{T}$, it follows that $g\left(a^{\alpha}\right)=1$ holds for all $a \in \tilde{T}$ and $\alpha \in \mathbb{B}^{S(a)}$. This implies that $g$ is a robust extension of the pBmb $(\tilde{T}, \tilde{F})$. Finally, since $g$ contains only robust terms, it is a very robust extension of $(\tilde{T}, \tilde{F})$.

Corollary 4. Problems $\operatorname{RE}(\mathcal{C})$ and $\operatorname{VR}(\mathcal{C})$ are equivalent for $\mathcal{C}=\mathcal{C}_{\text {all }}, \mathcal{C}_{\geq_{b}}$ with $b \in \mathbb{B}^{n}$ (thus in particular $\left.\mathcal{C}_{+}\right), \mathcal{C}_{2-D N F}, \mathcal{C}_{H o r n}, \mathcal{C}_{r-H o r n}, \mathcal{C}_{\text {unate }}, \mathcal{C}_{q-H o r n}$ and $\mathcal{C}_{D-\text { minor }}$.

The next corollary follows from Corollaries 3 and 4.

Corollary 5. For the classes $\mathcal{C}=\mathcal{C}_{\text {all }}, \mathcal{C}_{H o r n}, \mathcal{C}_{+}$, and $\mathcal{C}_{2-D N F}$, problems $\operatorname{VR}(\mathcal{C})$, $\mathrm{RE}(\mathcal{C})$ and $\mathrm{FC}(\mathcal{C})$ are all equivalent.

Besides Theorem [6] we have the following complexity results from Corollary 4 and the results of $\operatorname{RE}(\mathcal{C})$ in $[9]$.

Theorem 8. Problem $\operatorname{VR}(\mathcal{C})$ is polynomially solvable for $\mathcal{C}=\mathcal{C}_{D \text {-minor }}$, while it is $\mathrm{NP}$-hard for $\mathcal{C}=\mathcal{C}_{r-H o r n}$ and $\mathcal{C}_{\text {unate }}$.

\section{Cases of Non-equivalence between $\mathrm{FC}(\mathcal{C}), \operatorname{RE}(\mathcal{C})$, and $\operatorname{VR}(\mathcal{C})$}

In this section we shall show that problems $\mathrm{FC}(\mathcal{C}), \operatorname{RE}(\mathcal{C})$ and $\operatorname{VR}(\mathcal{C})$ are not always equivalent, despite the many quite general equivalences we have shown in the previous sections. We first give several classes $\mathcal{C}$ for which $\mathrm{FC}(\mathcal{C})$ and $\operatorname{RE}(\mathcal{C})$ are not equivalent, followed by a class for which $\operatorname{RE}(\mathcal{C})$ and $\operatorname{VR}(\mathcal{C})$ are not equivalent.

First, one might think that Theorem 1 could be generalized to prove the equivalence of $\operatorname{RE}(\mathcal{C})$ and $\mathrm{FC}(\mathcal{C})$ for classes closed under conjunction (but not necessarily closed under disjunction). This, however, is not the case, as the following simple example shows. Let us consider the class $\mathcal{C}^{*}$ consisting of functions $f$ for which $f(v) f(w)=0$ holds for all pairs of vectors $v, w \in \mathbb{B}^{n}$ which are at Hamming distance 1 . Clearly, this class $\mathcal{C}^{*}$ is closed under conjunction. Let 
us now consider the pBmb $(\tilde{T}, \tilde{F})$ given by $\tilde{T}=\{(1, *)\}$ and $\tilde{F}=\emptyset$. Since the equation $f(1,0)=f(1,1)=1$ must hold for any robust extension $f$ of $(\tilde{T}, \tilde{F}), f$ does not belong to $\mathcal{C}^{*}$. Therefore, $(\tilde{T}, \tilde{F})$ has no robust extension in $\mathcal{C}^{*}$. However, $f=x_{1} x_{2} \in \mathcal{C}^{*}$ is an extension of the pdBf $(\{(1,1)\}, \emptyset)$ and $g=x_{1} \bar{x}_{2} \in \mathcal{C}^{*}$ is an extension of the pdBf $(\{(1,0)\}, \emptyset)$. These imply that $(\tilde{T}, \tilde{F})$ is fully consistent with $\mathcal{C}^{*}$.

Let us demonstrate next that, for the class of threshold functions $\mathcal{C}_{T H}$, problems $\operatorname{RE}(\mathcal{C})$ and $\operatorname{FC}(\mathcal{C})$ are not equivalent. Let us recall first that a $\mathrm{pdBf}$ $(T, F)$ has a threshold extension, if and only if there exist $n+1$ real numbers $w_{1}, w_{2}, \ldots, w_{n}$ and $w_{0}$ such that:

$$
\sum_{j=1}^{n} w_{j} a_{j} \geq w_{0} \text { for all } a \in T \text {, textand } \sum_{j=1}^{n} w_{j} b_{j}<w_{0} \text { for all } b \in F .
$$

It is well known that this condition is also equivalent to the disjointness of their respective convex hulls,

$$
\operatorname{conv}(T) \cap \operatorname{conv}(F)=\emptyset,
$$

where $\operatorname{conv}(X)$ denotes the convex hull of the set $X$ in the $n$-dimensional real space. It is also easy to see by the definitions that a $\mathrm{pBmb}(\tilde{T}, \tilde{F})$ has a robust threshold extension if and only if

$$
\operatorname{conv}(\tilde{T}) \cap \operatorname{conv}(\tilde{F})=\emptyset,
$$

where $\operatorname{conv}(\tilde{X})=\operatorname{conv}\left(\cup_{\alpha \in \mathbb{B}^{S(\tilde{X})}} \tilde{X}^{\alpha}\right)$ for a subset $\tilde{X} \subseteq \mathbb{M}^{n}$.

Let us now consider the $\mathrm{pBmb}(\tilde{T}, \tilde{F})$ defined by

$$
\tilde{T}=\left\{\begin{array}{l}
(*, 1,1,1) \\
(0,0,0,0)
\end{array}\right\}, \quad \tilde{F}=\left\{\begin{array}{l}
(1,1,1,0) \\
(0,1,0,1) \\
(0,0,1,1)
\end{array}\right\} .
$$

The only one missing bit of $(\tilde{T}, \tilde{F})$ has two possible interpretations, yielding $T^{1}=\{(1,1,1,1),(0,0,0,0)\}$ and $\left.T^{0}=\{0,1,1,1),(0,0,0,0)\right\}$. It is easy to verify that the threshold Boolean function defined by $5 x_{1}-3 x_{2}-3 x_{3}+2 x_{4} \geq 0$ is an extension of the pdBf $\left(T^{1}, \tilde{F}\right)$, and that $-5 x_{1}+2 x_{2}+2 x_{3}-3 x_{4} \geq 0$ defines a threshold extension of $\left(T^{0}, \tilde{F}\right)$. Hence, the pBmb $(\tilde{T}, \tilde{F})$ is fully consistent with $\mathcal{C}_{T H}$. However, $(\tilde{T}, \tilde{F})$ has no robust threshold extension by (10), since the fractional vector $\left(\frac{1}{3}, \frac{2}{3}, \frac{2}{3}, \frac{2}{3}\right)$ belongs to the convex hulls of both $\tilde{T}$ and $\tilde{F}$.

We can also show the non-equivalence of $\operatorname{FC}(\mathcal{C})$ and $\operatorname{RE}(\mathcal{C})$ for the classes such as $\mathcal{C}_{\text {unate }}$ and $\mathcal{C}_{r-H o r n}$.

In concluding this section, we demonstrate with the example $(\tilde{T}, \tilde{F})$ defined in the table below that $\operatorname{RE}\left(\mathcal{C}_{3-D N F}\right)$ and $\operatorname{VR}\left(\mathcal{C}_{3-D N F}\right)$ are not equivalent.

$$
\tilde{T}=\{(1,1, *, 1,1)\}, \quad \tilde{F}=\left\{\begin{array}{l}
(1,1,0,1,0) \\
(1,1,0,0,1) \\
(1,0,1,1,1) \\
(0,1,1,1,1)
\end{array}\right\} \text {. }
$$


This pBmb does not have a very robust 3 -DNF extension, because the only robust term for $a=(1,1, *, 1,1)$ is the quartic term $t=x_{1} x_{2} x_{4} x_{5}$.

On the other hand, the $3-\mathrm{DNF}$

$$
\phi=x_{1} x_{2} x_{3} \vee \bar{x}_{3} x_{4} x_{5}
$$

is a robust extension of $(\tilde{T}, \tilde{F})$, hence $\mathcal{E}(\tilde{T}, \tilde{F}) \cap \mathcal{C}_{3-D N F} \neq \emptyset$.

Let us remark that in fact, problem $\operatorname{VR}\left(\mathcal{C}_{3-D N F}\right)$ is always polynomially decidable, while $\operatorname{RE}\left(\mathcal{C}_{3-D N F}\right)$ is co-NP-complete (see e.g., [9]).

\section{Complexity of $\mathrm{FC}\left(\mathcal{C}_{T H}\right)$}

We have already seen in Section 6 that problems $\operatorname{RE}\left(\mathcal{C}_{T H}\right)$ and $\operatorname{FC}\left(\mathcal{C}_{T H}\right)$ are not equivalent. It is known that $\operatorname{RE}\left(\mathcal{C}_{T H}\right)$ is polynomially solvable (see e.g. 9]), and we can show below that problem $\mathrm{FC}\left(\mathcal{C}_{T H}\right)$ is not only inequivalent, but has in fact a different complexity.

Theorem 9. Problem $\mathrm{FC}\left(\mathcal{C}_{T H}\right)$ is co-NP-complete, even if $|S(a)| \leq 1$ holds for all $a \in \tilde{T} \cup \tilde{F}$.

Proof. First we show that $\mathrm{FC}\left(\mathcal{C}_{T H}\right)$ belongs to co-NP. By (9), a pBmb $(\tilde{T}, \tilde{F})$ is not fully consistent with the class $\mathcal{C}_{T H}$ if and only if there exists an assignment $\alpha \in \mathbb{B}^{S}$ such that $\operatorname{conv}\left(\tilde{T}^{\alpha}\right) \cap \operatorname{conv}\left(\tilde{F}^{\alpha}\right) \neq \emptyset$. Therefore, $\mathrm{FC}\left(\mathcal{C}_{T H}\right)$ is in co-NP, since the last condition can be checked in polynomial time (for instance by linear programming).

To prove the completeness, we reduce the following NP-complete problem to our problem (see e.g., [12]).

\section{Problem ExACT COVER}

Input: $\quad$ A hypergraph $\mathcal{H}=(V, H)$ such that $V=\{1,2, \ldots, n\}$ and $H=\left\{E_{1}, E_{2}, \ldots, E_{m}\right\}$, where $E \subseteq V$ for all $E \in H$.

Question: Is there an $H^{*} \subseteq H$ which exactly covers $V$; i.e., for which $E \cap E^{\prime}=\emptyset$ for all $E \neq E^{\prime} \in H^{*}$ and $\bigcup_{E \in H^{*}} E=V$ ?

We may assume without loss of generality that any $H^{*}$ which exactly covers $V$ contains $E_{1}$. This does not affect the NP-hardness of the problem, as it can be seen easily, since we always can modify the input by including one more hyperedge, $E_{1}$, which is disjoint from all other hyperedges of $H$.

Let $V_{1}=\{n+1, n+2, \ldots, n+m\}$ and $V_{2}=\{n+m+1, n+m+2, \ldots, n+2 m\}$ and let $W=V \cup V_{1} \cup V_{2}$. We shall denote by $(R ; S)$ the vector $v \in \mathbb{M}^{W}$ for which $O N(v)=R$ and $S(v)=\{(v, j) \mid j \in S\}$. (Then $O F F(v)=V \backslash(R \cup S)$; thus in particular, $v=(R ; \emptyset)$ denotes a binary vector.) Let us define a $\operatorname{pBmb}(\tilde{T}, \tilde{F})$ by the following $\tilde{T}, \tilde{F} \subseteq \mathbb{M}^{W}$.

$$
\begin{aligned}
\tilde{T}= & \left\{a_{(1)}=(V \cup\{n+1\} \cup\{n+m+1\} ; \emptyset)\right\} \\
& \cup\left\{a_{(i)}=(\{n+m+i\} ;\{n+i\}), i=2,3, \ldots, m\right\} \\
\tilde{F}= & \left\{b_{(0)}=(\emptyset ; \emptyset)\right\} \cup\left\{b_{(1)}=\left(E_{1} \cup\{n+1\} \cup V_{2} ; \emptyset\right)\right\} \\
& \cup\left\{b_{(i)}=\left(E_{i} \cup\{n+i\} ; \emptyset\right), i=2,3, \ldots, m\right\} .
\end{aligned}
$$


For this pBmb we have $|S(a)| \leq 1$ for all $a \in \tilde{T}$ and $S(\tilde{F})=\emptyset$. Thus, we write simply $\tilde{F}$ instead of $\tilde{F}^{\alpha}$, in the sequel.

We claim that this $(\tilde{T}, \tilde{F})$ is not fully consistent with $\mathcal{C}_{T H}$ if and only if an exact cover $H^{*} \subseteq H$ exists. This will then imply the theorem.

First, we show the "only-if" part of the above claim. Let us assume that for an assignment $\alpha \in \mathbb{B}^{S(\tilde{T})}$ the pdBf $\left(\tilde{T}^{\alpha}, \tilde{F}\right)$ has no threshold extension. It follows from (9) that there exist nonnegative real numbers $\theta_{i}(i=1,2, \ldots, m)$ and $\eta_{i}$ $(i=0,1, \ldots m)$ such that

$$
\sum_{i=1}^{m} \theta_{i}=1, \quad \sum_{i=0}^{m} \eta_{i}=1, \quad \text { and } \quad \sum_{i=1}^{m} \theta_{i} a_{(i)}^{\alpha}=\sum_{i=0}^{m} \eta_{i} b_{(i)} .
$$

By comparing the corresponding components on the two sides of the last equality of (11), we have

$$
\begin{gathered}
\eta_{1}=\theta_{i}=\frac{1}{m} \text { for } i=1,2, \ldots, m \\
\eta_{i}=\left\{\begin{array}{l}
\frac{1}{m} \text { if } \alpha\left(a_{(i)}, n+i\right)=1, \\
0 \text { if } \alpha\left(a_{(i)}, n+i\right)=0 .
\end{array} \text { for } i=2,3, \ldots, m\right.
\end{gathered}
$$

Moreover, $\eta_{0}=1-\sum_{i=1}^{m} \eta_{i} \geq 0$ follows.

Let us define now a family $H^{*} \subseteq H$ by

$$
H^{*}=\left\{E_{i} \mid \eta_{i}=\frac{1}{m}, i=1,2, \ldots, m\right\}
$$

Although the proof is omitted (see 10), we can prove that $H^{*}$ is an exact cover of $H$, which completes the "only-if" part of our claim.

For the "if" part, take an arbitrary exact cover $H^{*} \subseteq H$, and associate an assignment $\alpha \in \tilde{F}$ to it by defining

$$
\alpha\left(a_{(i)}, n+i\right)= \begin{cases}1 & \text { if } E_{i} \in H^{*} \\ 0 & \text { otherwise }\end{cases}
$$

It is then easy to see that with the nonnegative real numbers

$$
\begin{aligned}
\theta_{i} & =\frac{1}{m}, \text { for } i=1,2, \ldots, m, \\
\eta_{i} & =\left\{\begin{array}{l}
\frac{1}{m} \text { if } E_{i} \in H^{*}, \\
0 \text { otherwise, }
\end{array} \text { for } i=1,2, \ldots, m,\right.
\end{aligned}
$$

and $\eta_{0}=1-\frac{\left|H^{*}\right|}{m}$, all equations in (11) hold. Hence $\tilde{T}^{\alpha}$ and $\tilde{F}$ are not linearly separable, which proves that $(\tilde{T}, \tilde{F})$ is not fully consistent with $\mathcal{C}_{T H}$. 


\section{Conclusion}

In this paper, we have considered the relations between problems $\operatorname{FC}(\mathcal{C}), \operatorname{RE}(\mathcal{C})$ and $\operatorname{VR}(\mathcal{C})$. We showed that for many general and reasonable families of classes such as $\mathcal{C}=\mathcal{C}_{\text {all }}, \mathcal{C}_{\text {Horn }}, \mathcal{C}_{+}$, and $\mathcal{C}_{2-D N F}$, these three problems are equivalent. We also demonstrated that such an equivalence does not hold for all classes $\mathcal{C}$. For instance, we showed that problem $\operatorname{RE}\left(\mathcal{C}_{T H}\right)$ is polynomially solvable, while $\mathrm{FC}\left(\mathcal{C}_{T H}\right)$ is co-NP-complete.

\section{References}

1. M. Anthony and N. Biggs. Computational Learning Theory. Cambridge University Press, 1992.

2. J.C. Bioch and T. Ibaraki. Lattice theoretic approaches to generalized monotone boolean functions and partially defined boolean functions. Manuscript, February 2000.

3. E. Boros, Y. Crama, and P.L. Hammer. Polynomial time inference of all valid implications for Horn and related formulae. Annals of Mathematics and Artificial Intelligence, 1:21-32, 1990.

4. E. Boros, V. Gurvich, P.L. Hammer, T. Ibaraki, and A. Kogan. Decompositions of partially defined Boolean functions. Discrete Applied Mathematics, 62:51-75, 1995.

5. E. Boros, P.L. Hammer, T. Ibaraki, and A. Kogan. Logical analysis of numerical data. Mathematical Programming, 79:163-190, August 1997.

6. E. Boros, P.L. Hammer, T. Ibaraki, A. Kogan, E. Mayoraz, and I. Muchnik. An implementation of logical analysis of data. IEEE Transactions on Knowledge and Data Engineering, to appear.

7. E. Boros, T. Ibaraki, and K. Makino. Monotone extensions of Boolean data sets. In M. Li and A. Maruoka, editors, Algorithmic Learning Theory - ALT'97, volume 1316 of Lecture Notes in Artificial Intelligence, pages 161-175, Berlin - New York, 1997. Springer Verlag.

8. E. Boros, T. Ibaraki, and K. Makino. Error-free and best-fit extensions of partially defined Boolean functions. Information and Computation, 140:254-283, 1998.

9. E. Boros, T. Ibaraki, and K. Makino. Logical analysis of binary data with missing bits. Artificial Intelligence, 107:219-263, 1999.

10. E. Boros, T. Ibaraki, and K. Makino. Fully consistent extensions of partially defined Boolean functions with missing bits. RUTCOR Research Report 9-99, Rutgers University, 640 Bartholomew Road, Piscataway, NJ 08854-8003, USA, 1999 (http://rutcor.rutgers.edu/ ${ }^{\text {rrr } / 1999 . h t m l) . ~}$

11. Y. Crama, P.L. Hammer, and T. Ibaraki. Cause-effect relationships and partially defined Boolean functions. Annals of Operations Research, 16:299-326, 1988.

12. M.R. Garey and D.S. Johnson. Computers and Intractability. Freeman, New York, NY, 1979.

13. K. Makino, K. Hatanaka, and T. Ibaraki. Horn extensions of a partially defined Boolean function. SIAM Journal on Computing, 28:2168-2186, 1999.

14. W. Quine. A way to simplify truth functions. American Mathematical Monthly, 62:627-631, 1955.

15. S. Rudeanu. Boolean Functions and Equations. North-Holland, Amsterdam, 1974. 